\title{
Banach fixed-point theorem in semilinear controllability problems - a survey
}

\author{
J. KLAMKA, A. BABIARZ*, and M. NIEZABITOWSKI \\ Institute of Automatic Control, Silesian University of Technology, 16 Akademicka St., 44-100 Gliwice, Poland
}

\begin{abstract}
The main aim of this article is to review the existing state of art concerning the complete controllability of semilinear dynamical systems. The study focus on obtaining the sufficient conditions for the complete controllability for various systems using the Banach fixedpoint theorem. We describe the results for stochastic semilinear functional integro-differential system, stochastic partial differential equations with finite delays, semilinear functional equations, a stochastic semilinear system, a impulsive stochastic integro-differential system, semilinear stochastic impulsive systems, an impulsive neutral functional evolution integro-differential system and a nonlinear stochastic neutral impulsive system. Finally, two examples are presented.
\end{abstract}

Key words: complete controllability, Banach space, Hilbert space, fixed-point, infinite-dimensional space.

\section{Introduction}

Controllability is one of the fundamental concepts in mathematical control theory and plays very important role both in stochastic and deterministic control systems [1,2].

We say that control system is controllable if each state corresponding to this process can be in appropriate time controlled or affected by some control signals. For finitedimensional systems, the notion of controllability was introduced in [3]. A few years later this notion was extended to infinite-dimensional systems $[4,5]$. Over the last decade the controllability has been extensively studied both for finite and infinite dimensional systems [6-18]. In the case of finitedimensional systems notions of complete and approximate controllability coincide. In infinite-dimensional spaces there exist linear subspaces, which are not closed, so we can distinguish concepts of the approximate and complete controllability. The approximate controllability means that system can be steered to an arbitrarily small neighbourhood of the final state. The complete controllability enables to steer the system to an arbitrary final state [19]. It means that complete controllability is fundamentally stronger notion, than approximate controllability.

The controllability of nonlinear deterministic systems in finite-dimensional space has been extensively studied, see [20-23] and references therein. The sufficient conditions for controllability of nonlinear systems in infinite-dimensional spaces may be found in [7-10, 24-27].

Dynamical systems with distributed delays in control were also considered in the literature $[28,29]$. The sufficient conditions for constrained controllability have been obtained and proved with some mapping theorems taken from functional analysis and linear approximation methods.

Controllability of nonlinear stochastic differential systems were discussed in [30-37]. Controllability of linear stochas- tic systems in finite-dimensional spaces is studied in [38-44]. Different types of controllability concepts for linear stochastic evolution equations can be found in papers $[45,46]$.

A controllability theory for abstract linear control systems in infinite-dimensional spaces was studied in many papers and monographs, see for example [47] and [48]. These concepts have been extended to infinite-dimensional systems represented by nonlinear evolution equations [49-51]. Most of the controllability results for nonlinear infinite-dimensional control systems concern the so-called semilinear control system that consists of a linear part and a nonlinear part.

Stochastic partial functional differential equations with finite delays are used to present stochastic models of biological, chemical and physical systems. The qualitative properties, for example stability, observability, controllability of these systems have not been studied in detail [52-54]. There exist literature on the related topics for deterministic partial differential equations with finite delays, see for example $[55,56]$. Problem of controllability for stochastic differential equations have been investigated in $[57,58]$.

The impulsive differential equations provide a natural description of observed evolutionary processes, which are subject to short term perturbations acting instantaneously in the form of impulses. Uncertainty can be incorporated either as an expression of our lack of precise knowledge or as a true driving force. In the latter case it is useful to model the system by a stochastic or noise driven model which leads to the study of stochastic impulsive differential systems.

The controllability for deterministic impulsive systems has been studied in $[59,60]$. The authors of [61] investigated the complete controllability of hybrid impulsive integrodifferential systems. In papers $[62,63]$ the necessary and sufficient conditions for state controllability and observability for a class of linear time-varying impulsive systems were

*e-mail: Artur.Babiarz@polsl.pl 
J. Klamka, A. Babiarz, and M. Niezabitowski

considered. In paper [64], the notion of complete controllability for nonlinear stochastic neutral impulsive systems in finite-dimensional spaces is introduced. Moreover, in this paper the sufficient conditions ensuring the complete controllability of the nonlinear stochastic impulsive system using the Banach fixed point theorem are established. The controllability of impulsive functional differential systems with infinite delay in Banach spaces was discussed in [65]. In paper [66] a set of sufficient conditions for complete controllability of impulsive neutral functional evolution integrodifferential systems in abstract spaces by using fixed point technique is established. Complete controllability of impulsive stochastic integro-differential systems was investigated in [67]. The controllability of switched impulsive control systems was discussed in [68]. The authors of [69] investigate the controllability of the first-order impulsive functional differential systems in Banach space. The controllability problem for a class of controlled switching impulsive systems was discussed in [70]. The authors of [71] formulate the sufficient conditions for the complete controllability of the second-order nonlinear impulsive control differential systems.

\section{Preliminaries}

2.1. Basic notations. In this paper we adopt the following notations:

- $H, K$ and $U$ are Hilbert spaces and $K$ and $U$ are separable;

- $\mathcal{L}(K, H)$ is the space of all bounded operators from $K$ to $H$;

- for $\psi \in \mathcal{L}(K, H)$ denote by $\psi^{*} \in \mathcal{L}(H, K)$ the adjoint operator;

- $(e(n))_{n \in \mathbb{N}}$ is a complete orthonormal basis in $K$;

- $A$ is a closed densely defined operator generating an analytic semigroup $\{S(t) ; t>0\}$ on $H$ with inner product $\langle\cdot, \cdot\rangle$ and norm $\|\cdot\|$;

- $A^{\alpha}: H_{\alpha} \subset H \rightarrow H$ is the fractional power operator with domain $H_{\alpha}$;

- in $H_{\alpha}$ we define the norm $\|x\|_{\alpha}:=\left\|A^{\alpha} x\right\|$ for $x \in H_{\alpha}$ (with this norm $H_{\alpha}$ is a Banach space (see [72]);

- $\mathcal{C}_{\alpha}=\mathcal{C}\left([-r, 0], H_{\alpha}\right)$ is the space of all continuous functions from $[-r, 0]$ into $H_{\alpha}, 0<r<\infty$;

- $B$ is a bounded linear operator from $U$ into $H$;

- $(\Omega, \mathcal{F}, P)$ is a probability space with probability measure $P$ on $\Omega$;

- $\mathbf{E}$ is expected value;

- $L_{p}(\Omega, H)$ is a space of all functions $V: \Omega \rightarrow H$ such that $\mathbf{E}\|V\|^{p}<\infty$

- $\left\{\mathcal{F}_{t}: t \geq 0\right\}$ is an increasing and right continuous family of complete sub $-\sigma-$ algebras of $\mathcal{F}$;

- $\phi$ is $\mathcal{F}_{0}-$ measurable stochastic process;

- if $T>0, \mathcal{X}$ - metric space and $F: \Omega \rightarrow \mathcal{X}$, then $F$ is called $\mathcal{F}_{t}$ - adapted if $F$ is $\mathcal{F}_{t}$ - measurable for almost all $t \in[0, T]$, and is called $\mathcal{F}_{0}-$ adapted if it is $\mathcal{F}_{0}-$ measurable for almost all $t \in[-r, 0]$;

- $X(t): \Omega \rightarrow H_{\alpha}, t \geq-r$, is a continuous $\mathcal{F}_{t}$ - adapted, $H_{\alpha}$ - valued stochastic process;
- $X_{t}: \Omega \rightarrow \mathcal{C}_{\alpha}, t \leq 0$ is defined by

$$
X_{t}(\omega)=\{X(t+s)(\omega): s \in[-r, 0]\}
$$

and it is called $\mathcal{C}_{\alpha}$ - valued stochastic process;

- $\left(\beta_{n}(t)\right)_{n \in \mathbb{N}}$ is the sequence of real-valued one-dimensional standard Brownian motions mutually independent over $(\Omega, \mathcal{F}, P)$

- for a sequence $\left(\lambda_{n}\right)_{n \in \mathbb{N}}, \lambda_{n} \geq 0$ we define

$$
W(t)=\sum_{n=1}^{\infty} \sqrt{\lambda_{n}} \beta_{n}(t) e_{n}, t \geq 0,
$$

then $W(t)$ is a $K$ - valued Wiener process with a finite trace nuclear covariance operator $Q \geq 0$, where $Q \in$ $\mathcal{L}(K, K)$ is the operator with the property $Q e_{n}=\lambda_{n} e_{n}$ and a finite trace

$$
\operatorname{tr} Q=\sum_{n=1}^{\infty} \lambda_{n}<\infty
$$

- $\mathcal{F}_{t}=\sigma(W(s): 0 \leq s \leq t)$ is the $\sigma$ - algebra generated by $W$ and $\mathcal{F}_{t}=\mathcal{F}$

- let $\psi \in \mathcal{L}(K, H)$ and define

$$
\|\psi\|_{Q}^{2}=\operatorname{tr}\left[\psi Q \psi^{*}\right]=\sum_{n=1}^{\infty}\left\|\sqrt{\lambda_{n}} \psi e_{n}\right\|^{2} ;
$$

- $\psi$ is a $Q$ - Hilbert-Schmidt operator if $\|\psi\|_{Q}<\infty$;

- $L_{2}^{0}(K, H)$ is the space of all $Q$ - Hilbert Schmidt operators from $K$ into $H$;

-

$$
\begin{gathered}
f:[0, \infty) \times \mathcal{C}_{\alpha} \times H \rightarrow H, \\
\sigma:[0, \infty) \times \mathcal{C}_{\alpha} \times H \rightarrow L_{2}^{0}(K, H)
\end{gathered}
$$

and

$$
g:[0, \infty) \times[0, \infty) \times \mathcal{C}_{\alpha} \rightarrow H
$$

are measurable mappings such that $f(t, 0,0), \sigma(t, 0,0)$ and $g(t, s, 0)$ are locally bounded in $H$ - norm, $L_{2}^{0}(K, H)-$ norm and $H$ - norm, respectively;

- $M \mathcal{C}_{\alpha}(0, p), p>2$ is the space of all $\mathcal{F}_{0}$ - measurable $\mathcal{C}_{\alpha}$ - valued functions $\varsigma: \Omega \rightarrow \mathcal{C}_{\alpha}$ with the norm

$$
\mathbf{E}\|\varsigma\|_{\mathcal{C}_{\alpha}}^{p}=\mathbf{E}\left\{\sup _{-r \leq s \leq 0}\left\|A^{\alpha} \varsigma(s)\right\|^{p}\right\}<\infty
$$

- $L_{p}^{\mathcal{F}}([0, T], H)$ is the closed subspace of

$$
L_{p}([0, T] \times \Omega \times \Omega, H)
$$

consisting of all $\mathcal{F}_{t}$ - adapted processes;

- $\mathcal{C}\left([-r, T], L_{p}(\Omega, H)\right)$ is the Banach space of all continuous maps from $[-r, T]$ into $L_{p}(\Omega, H)$ satisfying the condition

$$
\sup _{t \in[-r, T]} \mathbf{E}\|X(t)\|^{p}<\infty
$$

- $\mathcal{H}_{p}$ is the closed subspace of all continuous processes $X$ with trajectories in $\mathcal{C}\left([-r, T], L_{p}(\Omega, H)\right)$ with 
Banach fixed-point theorem in semilinear controllability problems - a survey

$$
\begin{gathered}
\|X\|_{\mathcal{H}_{p}}=\left(\sup _{t \in[0, T]} \mathbf{E}\left\|X_{t}\right\|_{\mathcal{C}}^{p}\right)^{1 / p} \\
=\left(\sup _{t \in[0, T]} \mathbf{E} \sup _{-r \leq s \leq 0}\left\|X_{t}(\omega)\right\|_{\mathcal{C}}^{p}\right)^{1 / p}<\infty .
\end{gathered}
$$

2.2. The Banach fixed-point theorem. Let us start with the following definition.

Definition 1 [47]. Let $(\mathcal{X}, d)$ be a metric space and $F: \mathcal{X} \rightarrow$ $\mathcal{X}$. We will say that operator $F$ is a contraction if there exists some $k \in(0,1)$ such that:

$$
\bigwedge_{x, y \in \mathcal{X}} d(F(x), F(y)) \leq k d(x, y) .
$$

Then, the Banach fixed-point theorem has a form:

Theorem 1 [47]. Let $\mathcal{X}$ be a Banach space and $F$ be a contraction on $\mathcal{X}$. Then, there exists a unique $x_{0} \in \mathcal{X}$ such that:

$$
F\left(x_{0}\right)=x_{0}
$$

\section{Complete controllability of semilinear systems}

In this section, we present dynamical systems described by various kind of semilinear abstract state equations.

\subsection{The stochastic semilinear functional integro-diffe-} rential system. In papers [73] and [74] the authors present sufficient conditions for the controllability of stochastic integro-differential systems in a finite-dimensional space. Two years later in paper [75] Balachandran, Park and Subalakshmi examine the complete controllability of the stochastic semilinear functional integro-differential system defined as follows

$$
\begin{gathered}
d X(t)=[-A X(t)+B u(t)] d t \\
+f\left(t, X_{t}, \int_{0}^{t} g\left(t, s, X_{s}\right) d s\right) d t \\
+\sigma\left(t, X_{t}, \int_{0}^{t} g\left(t, s, X_{s}\right) d s\right) d W(t), \\
t \in[0, T], \quad X_{0}=\phi \in L_{p}\left(\Omega, \mathcal{C}_{\alpha}\right) .
\end{gathered}
$$

The above-mentioned dynamical systems (1) have, the socalled mild solution, defined as follows:

Definition 2 [75]. A stochastic process $X$ is said to be a mild solution of the system (1) if the following conditions are satisfied:

1. $X(t, \omega)$ is measurable as a function from $[0, T] \times \Omega$ to $H$ and $X(t)$ is $\mathcal{F}_{t}$ - adapted;

2. $\mathbf{E}\|X(t)\|^{p}<\infty$ for each $t \in[-r, T]$;

3. for each $u \in L_{p}^{\mathcal{F}}([0, T], U)$ the process $X$ satisfies the following integral equation:

$$
\begin{gathered}
X(t)=S(t) \phi(0)+\int_{0}^{t} S(t-s) B u(s) d s \\
+\int_{0}^{t} S(t-s) f\left(s, X_{s}, \int_{0}^{s} g\left(s, \tau, X_{\tau}\right) d \tau\right) d s \\
+\int_{0}^{t} S(t-s) \sigma\left(s, X_{s}, \int_{0}^{s} g\left(s, \tau, X_{\tau}\right) d \tau\right) d W(s) \\
\quad \text { for } t \geq 0, \quad X_{0}=\phi \in M \mathcal{C}_{\alpha}(0, p),
\end{gathered}
$$

where $S(t)$ is an analytical semigroup.

The definition of the complete controllability of the stochastic semilinear functional integro-differential system has the following form:

Definition 3. System (1) is complete controllable $[0, T]$ if

$$
\mathcal{R}(t)=L_{p}(\Omega, \mathcal{F}, P, H),
$$

where

$$
\mathcal{R}(t)=\left\{X(t)=X(T ; u): u(\cdot) \in L_{p}^{\mathcal{F}}([0, T], U)\right\}
$$

is the reachable set at time $T$.

It means that all the points in $L_{p}(\Omega, \mathcal{F}, P, H)$ can be reached from initial state $\phi(0)$ at time $T$.

In order to define complete controllability, we should put some hypotheses [75]:

Hypothesis 1. The functions $f, \sigma$ and $g$ satisfy the following Lipschitz condition and for arbitrary $\gamma_{i}, \xi_{i} \in \mathcal{C}_{\alpha}, i=1,2$ and $0 \leq t \leq T$, suppose that there exist positive real constants $N_{1}, \widetilde{N}_{1}, K, \widetilde{K}>0$ such that

$$
\begin{gathered}
\left\|f\left(t, \gamma_{1}, \xi_{1}\right)-f\left(t, \gamma_{2}, \xi_{2}\right)\right\|^{p} \\
+\left\|\sigma\left(t, \gamma_{1}, \xi_{1}\right)-\sigma\left(t, \gamma_{2}, \xi_{2}\right)\right\|_{Q}^{p} \\
\leq N_{1}\left[\left\|\gamma_{1}-\gamma_{2}\right\|^{p}+\left\|\xi_{1}-\xi_{2}\right\|^{p}\right], \\
\|f(t, \gamma, \xi)\|^{p}+\|\sigma(t, \gamma, \xi)\|_{Q}^{p} \leq \widetilde{N}_{1}, \\
\left\|g\left(t, s, \gamma_{1}\right)-g\left(t, s, \gamma_{2}\right)\right\|^{p} \leq K\left\|\gamma_{1}-\gamma_{2}\right\|^{p}, \\
\|g(t, s, \xi)\|^{p} \leq \widetilde{K} .
\end{gathered}
$$

Hypothesis 2. The functions $f, \sigma$ and $g$ are continuous and satisfy the usual linear growth condition and for arbitrary $\gamma_{i}$, $\xi_{i} \in \mathcal{C}_{\alpha}, i=1,2$ and $t \in[0, T]$, suppose that there exist positive real constants $\widehat{N}_{1}, \widehat{K}>0$ such that

$$
\begin{gathered}
\|f(t, \gamma, \xi)\|^{p}+\|\sigma(t, \gamma, \xi)\|_{Q}^{p} \leq \widehat{N}_{1}\left(1+\|\gamma\|^{p}+\|\xi\|^{p}\right), \\
\|g(t, s, \xi)\|^{p} \leq \widehat{K}\left(1+\|\xi\|^{p}\right) .
\end{gathered}
$$

Hypothesis 3. $S(t), t \geq 0$ is the strongly continuous semigroup of bounded linear operators generated by operator $A$ and such that

$$
\max _{0 \leq t \leq T}\|S(t)\| \leq M
$$

where $M$ is the positive constant. 
Hypothesis 4. The linear operator $L_{0}^{T}$ from $L_{p}^{\mathcal{F}}([0, T], U)$ into $L_{p}(\Omega, \mathcal{F}, P, U)$, defined by

$$
L_{0}^{T}=\int_{0}^{T} S(t-s) B u(s) d s,
$$

induces a boundedly invertible operator $\widetilde{L}$ defined on

$$
L_{p}^{\mathcal{F}}([0, T], U) / \operatorname{ker} L_{0}^{T} .
$$

Now, we introduce operator $\Psi$, defined in the following way

$$
\begin{aligned}
& (\Psi Z)(t)=S(t) \phi(0)+\int_{0}^{t} S(t-s) B u(s, Z) d s \\
& +\int_{0}^{t} S(t-s) f\left(s, Z_{s}, \int_{0}^{s} g\left(s, \tau, Z_{\tau}\right) d \tau\right) d s \\
& +\int_{0}^{t} S(t-s) \sigma\left(s, Z_{s}, \int_{0}^{s} g\left(s, \tau, Z_{\tau}\right) d \tau\right) d W(s) .
\end{aligned}
$$

Under the Hypotheses 1-4, operator $\Psi$ has a fixed point $Z$ being the solution of the system (1), where the control process expressed as follows

$$
\begin{gathered}
u(t, Z)=\mathbf{E}\left\{(\widetilde{L})^{-1}(h-S(T) \phi(0)\right. \\
-\int_{0}^{T} S(T-s) f\left(s, Z_{s}, \int_{0}^{s} g\left(s, \tau, Z_{\tau}\right) d \tau\right) d s \\
\left.\left.-\int_{0}^{T} S(t-s) \sigma\left(s, Z_{s}, \int_{0}^{s} g\left(s, \tau, Z_{\tau}\right) d \tau\right) d W(s)\right) \mid \mathcal{F}_{t}\right\}
\end{gathered}
$$

is defined for an arbitrary process $Z_{s}$.

To formulate the main theorem of this subsection we have to introduce the next hypothesis:

Hypothesis 5. Let us use the basic notations from the Hypotheses $1-4$. Then the following inequality holds:

$$
\begin{gathered}
3^{p-1} M^{p} N_{5}\|B\|^{p} T^{p / q}+3^{p-1} M^{p} N_{1} T^{p / q}\left(1+K T^{p / q}\right) \\
+3^{p-1} M^{p} \frac{T^{p(\beta-1)+p / q}}{(q \beta-q+1)^{p / q}} \\
\cdot C_{p} \frac{T^{-p \beta+p / 2}}{(1-2 \beta)^{p / 2}} N_{1}\left(1+K T^{p / q}\right)<1,
\end{gathered}
$$

where

$$
\begin{gathered}
q=\frac{p}{p-1}, \quad \beta \in\left(\alpha, \frac{1}{2}\right), \\
C_{p}=\left(\frac{p}{2}(p-1)\right)^{p / 2}\left(\frac{p}{p-1}\right)^{p^{2} / 2}, \quad K>0
\end{gathered}
$$

and $N_{5}$ is the positive real constant such that for all $X, Y \in \mathcal{H}_{p}$

$$
\mathbf{E}\|u(t, X)-u(t, Y)\|^{p} \leq N_{5} \int_{0}^{T} \mathbf{E}\left\|X_{s}-Y_{s}\right\|_{\mathcal{C}_{\alpha}}^{p} d s .
$$

Theorem 2 [75]. Assume that Hypotheses 1-5 are satisfied. Then the system (1) is complete controllable on $[0, T]$.

In the proof of Theorem 2 the Banach fixed point theorem is used.

\subsection{The stochastic partial differential equations with fi-} nite delays. Let us consider the special case of the system (1), which is defined in Hilbert spaces and investigated in [76]. Substituting $g\left(t, s, X_{s}\right)=0$ in equation (1) we obtain the following state equation:

$$
\begin{gathered}
d X(t)=\left[-A X(t)+B u(t)+f\left(t, X_{t}\right)\right] d t \\
+\sigma\left(t, X_{t}\right) d W(t), \\
t \in[0, T], \quad X_{0}=\phi \in L_{p}\left(\Omega, \mathcal{C}_{\alpha}\right)
\end{gathered}
$$

for which almost all basic notations introduced in Subsec. 2.1 are valid. However, it should be pointed out, that there some essential differences:

- $f:[0, \infty) \times \mathcal{C}_{\alpha} \rightarrow H$ and $\sigma:[0, \infty) \times \mathcal{C}_{\alpha} \rightarrow L_{2}^{0}(K, H)$ are two measurable mappings such that $f(t, 0)$ and $\sigma(t, 0)$ are locally bounded in $H$ - norm and $L_{2}^{0}(K, H)$ - norm, respectively;

- $L_{p}^{\mathcal{F}}([0, T], H)$ is the closed subspace of $L_{p}([0, T] \times \Omega, H)$ consisting of $\mathcal{F}_{t}$ - adapted processes.

The mild solution of dynamical system described by state Eq. (2) is defined as follows:

Definition 4 [76]. A stochastic process $X$ is said to be a mild solution of the system (2) if the following conditions are satisfied:

1. $X(t, \omega)$ is measurable as a function from $[0, T] \times \Omega$ to $H$ and $X(t)$ is $\mathcal{F}_{t}$ - adapted;

2. $\mathbf{E}\|X(t)\|^{p}<\infty$ for each $t \in[-r, T]$;

3. for each $u \in L_{p}^{\mathcal{F}}([0, T], U)$ the process $X$ satisfies the following integral equation:

$$
\begin{gathered}
X(t)=S(t) \phi(0) \\
+\int_{0}^{t} S(t-s)\left(B u(s)+f\left(s, X_{s}\right)\right) d s \\
+\int_{0}^{t} S(t-s) \sigma\left(s, X_{s}\right) d W(s), \quad t \geq 0 \\
X_{0}=\phi \in M \mathcal{C}_{\alpha}(0, p) .
\end{gathered}
$$

As in the previous subsection, several hypotheses [76] are specified. 
Hypothesis 6. For arbitrary $\gamma, \xi \in \mathcal{C}_{\alpha}$ and $t \in[0, T]$, suppose that there exists a positive real constant $N_{1}>0$ such that

$$
\begin{gathered}
\|f(t, \gamma)-f(t, \xi)\|^{p}+\|\sigma(t, \gamma)-\sigma(t, \xi)\|_{Q}^{p} \leq N_{1}\|\gamma-\xi\|_{\mathcal{C}_{\alpha}}^{p} \\
\|f(t, \xi)\|^{p}+\|\sigma(t, \xi)\|_{Q}^{p} \leq N_{1}\left(1+\|\xi\|_{\mathcal{C}_{\alpha}}^{p}\right) .
\end{gathered}
$$

To formulate the main theorem devoted to complete controllability of the stochastic partial differential equations with finite delays, it is necessary to use Definition 3 and Hypotheses 3, 4. Moreover, under Hypotheses 3, 4 and 6, for an arbitrary process $Z_{s}$, the control process can be defined

$$
\begin{aligned}
u(t, Z)= & \mathbf{E}\left\{( \widetilde { L } ) ^ { - 1 } \left(h-S(T) \phi(0)-\int_{0}^{T} S(t-s) f\left(s, Z_{s}\right) d s\right.\right. \\
& \left.\left.-\int_{0}^{T} S(t-s) \sigma\left(s, Z_{s}\right) d W(s)\right) \mid \mathcal{F}_{t}\right\} .
\end{aligned}
$$

that guarantees, the nonlinear operator $\Psi$, defined by the following formula

$$
\begin{gathered}
(\Psi Z)(t)=S(t) \phi(0)+\int_{0}^{t} S(t-s) B u(s, Z) d s \\
+\int_{0}^{t} S(t-s) f\left(s, Z_{s}\right) d s+\int_{0}^{t} S(t-s) \sigma\left(s, Z_{s}\right) d W(s)
\end{gathered}
$$

it has a fixed point $Z$, which is a solution of (2).

Theorem 3 [76]. Assume that Hypotheses 3-6 are satisfied. Then the system (2) is completely controllable on $[0, T]$.

The proof of Theorem 3 can be found in [76] and it is based on the application of the contraction theorem.

3.3. The semilinear functional equations. Let $C([-h, 0]$, $X)$ shortly denoted as $C$ be the Banach space of all continuous functions from an interval $[-h, 0]$ to $X$ with the supremum norm. The authors of paper [77] study the complete controllability of dynamical systems given by the semilinear evolution equation

$$
\begin{gathered}
d x(t)=\left[A x(t)+B u(t)+f\left(t, x_{t}, u(t)\right)\right] d t, \\
x_{0}(\theta)=\phi(\theta), \quad \theta \in[-h, 0], \quad t \in(0, T],
\end{gathered}
$$

where

- the state $x(\cdot)$ takes values in a Hilbert space $X$;

- the control $u(\cdot) \in L_{2}([0, T], U)$ takes values in a Hilbert space $U$;

- $\phi \in C$.

If $x:[-h, T] \rightarrow X$ is a continuous function, then $x_{t}$ is an element in $C$ which has point-wise definition

$$
x_{t}(\theta)=x(t+\theta) \quad \text { for } \quad \theta \in[-h, 0] .
$$

The solution of system (3) can be expressed as the following form:

$$
\begin{gathered}
x_{t}(0)=x(t)=S(t) \phi(0) \\
+\int_{0}^{t} S(t-s)\left[B u(s)+f\left(s, x_{s}, u(s)\right)\right] d s, \\
x_{0}(\theta)=\phi(\theta), \quad \theta \in[-h, 0], \quad t \in(0, T],
\end{gathered}
$$

where $S(t)$ is a linear semigroup on $X, B: U \rightarrow X$ is a bounded linear operator.

Definition 5 [77]. System (3) is completely controllable on the interval $[0, T]$ if

$$
\mathcal{R}(T, \phi)=X,
$$

where

$$
\mathcal{R}(T, \phi)=\left\{x_{T}(\phi ; u)(0): u(\cdot) \in L_{2}([0, T], U)\right\}
$$

is the reachable set at time $T$. It means that all the points in $X$ can be reached from initial state $\phi$ at time $T$.

For simplicity of considerations let us introduce the following notations

$$
\begin{gathered}
K=\max \{\|S(t)\|: 0 \leq t \leq T\}, \\
M=\|B\| .
\end{gathered}
$$

Moreover, let us assume the following hypotheses [77]:

Hypothesis 7. The function $f:[0, T] \times C \times U \rightarrow X$ is continuous and there exists $L>0$ such that

$$
\|f(t, \phi, u)\| \leq L\left(1+\|\phi\|_{C}+\|u\|\right)
$$

for all $(t, \phi, u) \in[0, T] \times C \times U$.

Hypothesis 8. The function $f:[0, T] \times C \times U \rightarrow X$ satisfies the Lipschitz condition

$$
\begin{aligned}
& \left\|f\left(t, \phi_{1}, u_{1}\right)-f\left(t, \phi_{2}, u_{2}\right)\right\| \\
\leq & L\left(\left\|\phi_{1}-\phi_{2}\right\|_{C}+\left\|u_{1}-u_{2}\right\|\right) .
\end{aligned}
$$

Hypothesis 9. Let us introduce two crucial operators:

$$
\begin{gathered}
\Gamma_{0}^{T}=\int_{0}^{T} S(T-s) B B^{*} S^{*}(T-s) d s, \\
R\left(\alpha, \Gamma_{0}^{T}\right)=\left(\alpha I+\Gamma_{0}^{T}\right)^{-1} .
\end{gathered}
$$

Moreover, let us assume that $\alpha R\left(\alpha, \Gamma_{0}^{T}\right) \rightarrow 0$ as $\alpha \rightarrow 0^{+}$in the uniform operator topology.

Hypothesis 10. There exist $\gamma>0$ such that

$$
\left\langle\Gamma_{0}^{T} x, x\right\rangle \geq \gamma\|x\|^{2} \quad \text { for all } \quad x \in X .
$$

It means that $\Gamma_{0}^{T}$ is an invertible operator

$$
\left\|\left(\Gamma_{0}^{T}\right)^{-1}\right\| \leq \frac{1}{\gamma}
$$

Now, let us define the nonlinear operator $\mathbb{F}^{0}$ on

$$
C([0, T], C) \times C([0, T], U)
$$

as follows

$$
\mathbb{F}^{0}(x, u)=(z, \nu),
$$


where

$$
\begin{gathered}
\nu(t)=B^{*} S^{*}(T-t)\left(\Gamma_{0}^{T}\right)^{-1} p(x, u), \\
z(t)=S(t) \phi(\theta)+\int_{0}^{t} S(t-s)\left(B \nu(s)+f\left(s, x_{s}, u(s)\right)\right) d s, \\
z_{0}(\theta)=\phi(\theta), \quad \theta \in[-h, 0], \\
p(x, u)=x_{T}-S(t) \phi-\int_{0}^{T} S(t-s) f\left(s, x_{s}, u(s)\right) d s .
\end{gathered}
$$

Finally, the theorem about the complete controllability of dynamical system (3) has the following form:

Theorem 4 [77]. Assume that Hypotheses 7-10 hold. If

$$
\left(\frac{1}{\gamma} K^{2} M+\frac{1}{\gamma} K^{3} M^{2} T+K\right) T L<1,
$$

then the operator $\mathbb{F}^{0}$ has a unique fixed point in

$$
C([0, T], C) \times C([0, T], U)
$$

and the system (3) is completely controllable on $[0, T]$.

Similarly as before, proof is based on the Banach fixed point theorem.

3.4. Stochastic semilinear system. In paper [78] the complete controllability property of semilinear stochastic systems assuming controllability of the associated linear systems is studied. The stochastic linear system is defined by the following formula

$$
\begin{gathered}
d x(t)=[A x(t)+B u(t)] d t+\sigma(t) d W(t), \\
x(0)=x_{0}, \quad t \in[0, T]
\end{gathered}
$$

and the corresponding stochastic semilinear system is as follows

$$
\begin{gathered}
d x(t)=[A x(t)+B u(t)+F(t, x(t), u(t))] d t \\
+\sigma(t, x(t), u(t)) d W(t), \\
x(0)=x_{0}, \quad t \in[0, T],
\end{gathered}
$$

where

- $A: H \rightarrow H$ is an infinitesimal generator of strongly continuous semi-group $S(\cdot)$;

- $B \in \mathcal{L}(U, H)$;

- $F:[0, T] \times H \times U \rightarrow H$ and $\sigma:[0, T] \times H \times U \rightarrow L_{2}^{0}$.

The definition of complete controllability of stochastic linear system (6) is as follows.

Definition 6 [78]. System (6) is completely controllable on $[0, T]$ if

$$
\mathcal{R}_{T}\left(x_{0}\right)=L_{2}\left(\mathcal{F}_{T}, H\right),
$$

with the set of all states reachable from $x_{0}$ in time $t>0$ is defined as follows

$$
\mathcal{R}_{t}\left(x_{0}\right)=\left\{x\left(t ; x_{0}, u\right): u \in L_{2}^{\mathcal{F}}([0, T], H)\right\},
$$

where $x\left(t ; x_{0}, u\right)$ is the solution of system (6).
The solution of system (6) is defined by the solution of the nonlinear integral equation given by the form:

$$
\begin{gathered}
x(t)=S(t) x_{0}+\int_{0}^{t} S(t-s) \\
\cdot[B u(s)+F(s, x(s), u(s))] d s \\
+\int_{0}^{t} S(t-s) \sigma(s, x(s), u(s)) d W(s),
\end{gathered}
$$

where $U_{a d}$ is the space of admissible controls and $u \in U_{a d}:=$ $U_{2}$.

For convenience, it poses some hypotheses [78].

Hypothesis 11. $(F, \sigma):[0, T] \times H \times U \rightarrow H \times L_{2}^{0}$ satisfies the Lipschitz condition with respect to $(x, u)$ for all $t \in[0, T]$,

$$
\begin{gathered}
\left\|F\left(t, x_{1}, u_{1}\right)-F\left(t, x_{2}, u_{2}\right)\right\|^{2}+\left\|\sigma\left(t, x_{1}, u_{1}\right)-\sigma\left(t, x_{2}, u_{2}\right)\right\|^{2} \\
\leq L\left(\left\|x_{1}-x_{2}\right\|^{2}+\left\|u_{1}-u_{2}\right\|^{2}\right) .
\end{gathered}
$$

Hypothesis 12. $(F, \sigma)$ is continuous on $[0, T] \times H \times U$ and satisfies

$$
\|F(t, x, u)\|^{2}+\|\sigma(t, x, u)\|^{2} \leq L\left(1+\|x\|^{2}+\|u\|^{2}\right) .
$$

Hypothesis 13. The linear system (5) is completely controllable on $[0, T]$ if there exists $\gamma>0$ such that:

$$
\mathbf{E}\left\langle\Gamma_{0}^{T} z, z\right\rangle \geq \gamma \mathbf{E}\|z\|^{2} \text { for all } z \in L_{2}(\mathcal{F}, H) .
$$

In order to study the complete controllability we have to define the nonlinear operator $\Phi^{0}$ from $\mathcal{H}_{2} \times U_{a d}$ to $\mathcal{H}_{2} \times U_{a d}$ given by the following form:

$$
(z(t), \nu(t))=\Phi^{0}(x, u)(t)
$$

where

$$
\begin{aligned}
& z(t)= S(t) \\
& x_{0} \int_{0}^{t} S(t-s)[B \nu(s)+F(s, x(s), u(s))] d s \\
&+\int_{0}^{t} S(t-s) \sigma(s, x(s), u(s)) d W(s), \\
& \nu(t)=B^{*} S^{*}(T-t) \mathbf{E}\left\{( \Gamma _ { 0 } ^ { T } ) ^ { - 1 } \left(h-S(t) x_{0}\right.\right. \\
&-\int_{0}^{T} S(t-s) F(s, x(s), u(s)) d s \\
&\left.\left.-\int_{0}^{T} S(t-s) \sigma(s, x(s), u(s)) d W(s)\right) \mid \mathcal{F}_{t}\right\} .
\end{aligned}
$$

Hypothesis 14. The nonlinear operator $\Phi^{0}$ has a fixed point if formula:

$$
\left(\frac{2}{\gamma} l^{2} M(T+1)+\frac{4}{\gamma} l^{3} M^{2}(T+1) T+4 l T+4 l\right) T L<1
$$

is satisfied and $M=\|B\|^{2}, l=\max \left\{\|S(t)\|^{2}: t \in[0, T]\right\}$. 
Now, the theorem can be posed.

Theorem 5 [78]. Under Hypotheses 11-14 the system (6) is completely controllable on $[0, T]$.

The proof is obtained by using the Banach fixed-point theorem.

\section{Complete controllability of impulsive systems}

Moreover, in the literature the complete controllability is studied for impulsive systems. Different impulsive semilinear systems are the content of this section.

4.1. Impulsive stochastic integro-differential system. In [79], authors consider the complete controllability of following impulsive stochastic integro-differential systems in a Hilbert space:

$$
\begin{gathered}
d x(t)=[A x(t)+B u(t) \\
\left.+F\left(t, x(t), \int_{0}^{t} f(t, s, x(s)) d s\right)\right] d t \\
+G\left(t, x(t), \int_{0}^{t} g(t, s, x(s)) d s\right) d W(t), \\
t \neq t_{k}, t \geq 0, \Delta x\left(t_{k}\right)=I_{k}\left(x_{t_{k}^{-}}\right), \\
\quad k=1,2, \cdots, m, \quad x(0)=x_{0} \in H,
\end{gathered}
$$

where

- $F:[0, T] \times H \times H \rightarrow H$;

- $G:[0, T] \times H \times H \rightarrow \mathcal{L}_{2}\left(Q^{1 / 2} E, H\right)$;

- $f, g:[0, T] \times[0, T] \times H \rightarrow H$ are measurable mappings;

- $I_{k}\left(x_{t_{k}^{-}}\right)=x\left(t_{k}^{+}\right)-x\left(t_{k}^{-}\right), t=t_{k}, k=1,2, \cdots, \rho$, where $x\left(t_{k}^{+}\right)$and $x\left(t_{k}^{-}\right)$denote the right and left limits of $x(t)$ at $t=t_{k}$ respectively;

- $\Delta x\left(t_{k}\right)=x\left(t_{k}^{+}\right)-x\left(t_{k}^{-}\right)$represents the jump in the state $x$ at time $t_{k}$ with $I_{k}$ determining the size of the jump.

The mild solution of system (9) is given by the following nonlinear integral equation:

$$
\begin{gathered}
x(t)=S(t) x_{0}+\int_{0}^{t} S(t-s) B u(s) d s \\
+\int_{0}^{t} S(t-s) F\left(s, x(s), \int_{0}^{s} f(s, \tau, x(\tau)) d \tau\right) d s \\
+\int_{0}^{t} S(t-s) G\left(s, x(s), \int_{0}^{s} g(s, \tau, x(\tau)) d \tau\right) d W(s) \\
+\sum_{k=1}^{\rho} S\left(t-t_{k}\right) I_{k}\left(x\left(t_{k}^{-}\right)\right) .
\end{gathered}
$$

The definition of the impulsive stochastic integrodifferential systems in a Hilbert space (9) is the following.
Definition 7 [79]. The dynamical system (9) is completely controllable on $[0, T]$ if

$$
\mathcal{R}_{T}\left(x_{0}\right)=L_{2}^{\mathcal{F}_{T}}([0, T], H) .
$$

To formulate the main result of this subsection, i.e. theorem about complete controllability using the Banach fixedpoint theorem, we have to introduce the next lemma and a few hypotheses [79].

Lemma 1. Suppose that the controllability operator $\Gamma_{0}^{T} \in$ $\mathcal{L}(H, H)$ associated with linear dynamical systems defined as follows:

$$
\Gamma_{0}^{T}=\int_{0}^{T} S(T-t) B B^{*} S^{*}(T-t) d t
$$

is invertible. Then the control, for arbitrary target $x_{T} \in$ $L_{2}\left(\mathcal{F}_{T}, H\right)$, is defined by the following formula

$$
\begin{gathered}
u(t)=B^{*} S^{*}(T-t) \mathbf{E} \\
\times\left\{( \Gamma _ { 0 } ^ { T } ) ^ { - 1 } \left[x_{T}-S(T) x_{0}-\int_{0}^{T} S(T-s) \bar{F}(s) d s\right.\right. \\
-\int_{0}^{T} S(T-s) \bar{G}(s) d w(s) \\
\left.\left.-\sum_{k=1}^{\rho} S\left(T-t_{k}\right) I_{k}\left(x\left(t_{k}^{-}\right)\right)\right] \mid \mathcal{F}_{t}\right\}
\end{gathered}
$$

and steers the systems (10) from $x_{0}$ to $x_{T}$ at time $T$, where

$$
\begin{aligned}
& \bar{F}(s)=F\left(s, x(s), \int_{0}^{s} f(s, \tau, x(\tau)) d \tau,\right. \\
& \bar{G}(s)=G\left(s, x(s), \int_{0}^{s} g(s, \tau, x(\tau)) d \tau .\right.
\end{aligned}
$$

Hypothesis 15. The functions $F, G$ and $I$ are continuous and satisfy the usual linear growth condition; that is, there exist positive real constants $L_{1}, \alpha_{k}$ for arbitrary $x \in H$, and $t \in[0, T]$ such that:

$$
\begin{gathered}
\|F(t, x, y)\|^{2}+\|G(t, x, y)\|_{\mathcal{L}_{2}^{0}}^{2} \leq L_{1}\left(1+\|x\|^{2}+\|y\|^{2}\right), \\
\left\|I_{k}(x)\right\|^{2} \leq \alpha_{k}\left(1+\|x\|^{2}\right), \quad k=1,2, \ldots, \rho, \\
\left\|\int_{0}^{t} f(t, s, x(s)) d s\right\|^{2}+\left\|\int_{0}^{t} g(t, s, x(s)) d s\right\|^{2} \leq k_{1}\|x\|^{2} .
\end{gathered}
$$

Hypothesis 16. The functions $F, G$ and $I$ satisfy the following Lipschitz condition and for every $t \geq 0$ and $x, y \in H$ there exist positive real constants $L_{2}, \beta_{k}, k_{2}$ such that:

$$
\begin{gathered}
\left\|F\left(t, x_{1}, y_{1}\right)-F\left(t, x_{2}, y_{2}\right)\right\|^{2} \\
+\left\|G\left(t, x_{1}, y_{1}\right)-G\left(t, x_{2}, y_{2}\right)\right\|_{\mathcal{L}_{2}^{0}}^{2} \\
\leq L_{2}\left(\left\|x_{1}-x_{2}\right\|^{2}+\left\|y_{1}-y_{2}\right\|^{2}\right), \\
\left\|I_{k}(x)-I_{k}(y)\right\| \leq \beta_{k}\|x-y\|, \quad k=1,2, \ldots, \rho,
\end{gathered}
$$




$$
\begin{gathered}
\int_{0}^{t}\left\{\|f(t, s, x(s))-f(t, s, y(s))\|^{2}\right. \\
\left.+\int_{0}^{t}\|g(t, s, x, x(s))-g(t, s, y(s))\|^{2}\right\} d s \leq k_{2}\|x-y\|^{2} .
\end{gathered}
$$

Hypothesis 17. The given linear system:

$$
d x(t)=[A x(t)+B u(t)] d t+D(t) d W(t), \quad x(0)=x_{0}
$$

is completely controllable for some $\gamma>0$,

$$
\mathbf{E}\left\langle\Gamma_{0}^{T} z, z\right\rangle \geq \gamma \mathbf{E}\|z\|^{2}
$$

for all $z \in L_{2}\left(\mathcal{F}_{T}, H\right)$. Then,

$$
\left\|\left(\Gamma_{0}^{T}\right)^{-1}\right\| \leq \frac{1}{\gamma}=l_{2} .
$$

For our convenience, we introduce the following notations:

$$
l_{1}=\max _{t \in[0, T]}\|S(t)\|^{2}, \quad M=\max _{s \in[0, T]}\left\|\Gamma_{s}^{T}\right\|^{2} .
$$

Hypothesis 18. There exist positive real constants $l_{1}, l_{2}, \rho$, $M, L_{2}, \beta_{k}$ and $k_{2}$ such that

$$
\begin{gathered}
{\left[6 T l_{1} L_{2}\left(M l_{1} l_{2}+1\right)(T+4)\left(1+k_{2} T\right)\right.} \\
\left.\quad+6 l_{1} \rho\left(M l_{2}+1\right) \Sigma_{k=1}^{\rho} \beta_{k}\right]<1 .
\end{gathered}
$$

Now, it can be defined a nonlinear operator $\Phi$ from $H_{2}$ to $\mathrm{H}_{2}$ by the following form:

$$
\begin{gathered}
(\Phi x)(t)=S(t) x_{0}+\int_{0}^{t} S(t-s) B u(s) d s \\
+\int_{0}^{t} S(t-s) \bar{F}(s, x) d s+\int_{0}^{t} S(t-s) \bar{G}(s, x) d W(s) \\
+\sum_{k=1}^{\rho} S\left(t-t_{k}\right) I_{k}\left(x\left(t_{k}^{-}\right)\right),
\end{gathered}
$$

where $u(t)$ is defined by Eq. (12).

Then, the following theorem can be posed:

Theorem 6 [79]. Suppose that Hypotheses 15-18 are satisfied and the operator $\Phi$ is a contraction mapping from $\mathrm{H}_{2}$ to $\mathrm{H}_{2}$, and has a unique fixed point. Therefore, the dynamical system (10) is completely controllable on $[0, T]$.

4.2. Semilinear stochastic impulsive systems. The controllability of semilinear stochastic impulsive systems in Hilbert spaces is investigated in [80]. The authors used the Banach fixed-point theorem and Burkholder-Davis-Gundy inequality to obtain the sufficient conditions for the complete controllability. Authors of [80] consider the impulsive stochastic systems described by the following formula:

$$
\begin{gathered}
d x(t)=[A x(t)+B u(t)+F(t, x(t))] d t \\
+\sigma(t, x(t)) d W(t), \quad t \neq t_{k}, \quad t \geq 0, \\
\Delta x\left(t_{k}\right)=I_{k}\left(x_{t_{k}^{-}}\right), \quad t=t_{k}, \\
k=1,2, \ldots, \rho, \quad x_{0}(\cdot)=x_{0},
\end{gathered}
$$

where

- $A: H \rightarrow H$ is the linear unbounded operator;

- $B \in \mathcal{L}(U, H)$;

- $F:[0, T] \times H \rightarrow H$;

- $\Sigma:[0, T] \times H \rightarrow L_{2}^{0}$

The mild solution of system (14) is given by the following nonlinear integral equation:

$$
\begin{gathered}
x(t)=S(t) x_{0}+\int_{0}^{t} S(t-s) B u(s) d s \\
+\int_{0}^{t} S(t-s) F(s, x) d s+\int_{0}^{t} S(t-s) \sigma(s, x) d W(s) \\
+\sum_{k=1}^{\rho} S\left(t-t_{k}\right) I_{k}\left(x\left(t_{k}^{-}\right)\right) .
\end{gathered}
$$

In paper [80], the definition of complete controllability of semilinear stochastic impulsive systems in Hilbert spaces can be found (14), which is described by the Definition 7 .

To formulate the main result of this subsection, i.e. the theorem about complete controllability using the Banach fixedpoint theorem, we have to introduce a few hypotheses $[79,80]$. It should be pointed out that Hypotheses 20-22 are the simpler form of the Hypotheses 15-17.

Hypothesis 19. $A$ is the infinitesimal generator of strongly continuous semi-group $S(t)$ for $t \geq 0$.

Hypothesis 20. The functions $F, \sigma$ and $I$ (unit operator) are continuous and satisfy the usual linear growth condition; that is, there exist positive real constants $L_{1}, \alpha_{k}$ for arbitrary $x \in H$, and $t \in[0, T]$ such that:

$$
\begin{gathered}
\|F(t, x)\|^{2}+\|\sigma(t, x)\|_{\mathcal{L}_{2}^{0}}^{2} \leq L_{1}\left(1+\|x\|^{2}\right), \\
\left\|I_{k}(x)\right\|^{2} \leq \alpha_{k}\left(1+\|x\|^{2}\right), \quad k=1,2, \ldots, \rho .
\end{gathered}
$$

Hypothesis 21. The functions $F, \sigma$ and $I$ satisfy the following Lipschitz condition and for every $t \geq 0$ and $x, y \in H$ there exist positive real constants $L_{2}, \beta_{k}$ such that:

$$
\begin{gathered}
\|F(t, x)-F(t, y)\|^{2}+\|\sigma(t, x)-\sigma(t, y)\|_{\mathcal{L}_{2}^{0}}^{2} \\
\quad \leq L_{2}\|x-y\|^{2}, \\
\left\|I_{k}(x)-I_{k}(y)\right\| \leq \beta_{k}\|x-y\|, \quad k=1,2, \ldots, \rho .
\end{gathered}
$$

Hypothesis 22. The given linear system:

$$
d x(t)=[A x(t)+B u(t)] d t+D(t) d W(t), \quad x(0)=x_{0}
$$

is completely controllable for some $\gamma>0$,

$$
\mathbf{E}\left\langle\Gamma_{0}^{T} z, z\right\rangle \geq \gamma \mathbf{E}\|z\|^{2}
$$

for all $z \in L_{2}\left(\mathcal{F}_{T}, H\right)$. Then,

$$
\left\|\left(\Gamma_{0}^{T}\right)^{-1}\right\| \leq \frac{1}{\gamma}=l_{2} .
$$

For our convenience, we introduce the following notations:

$$
l_{1}=\max _{t \in[0, T]}\|S(t)\|^{2}, \quad M=\max _{s \in[0, T]}\left\|\Gamma_{s}^{T}\right\|^{2} .
$$




\section{Banach fixed-point theorem in semilinear controllability problems - a survey}

Then, the following theorem can be posed:

Theorem 7 [80]. Suppose that assumptions 19-22 hold. Then, the semilinear stochastic impulsive system (14) is completely controllable provided:

$$
\begin{gathered}
6 l_{1}\left[T L_{2}\left(M T l_{1} l_{2}+4 M l_{1} l_{2}+4 T+1\right)\right. \\
\left.+\rho \sum_{k=1}^{\rho} \beta_{k}\left(M l_{2}+1\right)\right]<1 .
\end{gathered}
$$

The proof of Theorem 7 contains the nonlinear operator described by

$$
\begin{gathered}
(\Phi x)(t)=S(t) x_{0}+\int_{0}^{t} S(t-s) B u(s) d s \\
+\int_{0}^{t} S(t-s) F(s, x) d s+\int_{0}^{t} S(t-s) \sigma(s, x) d W(s) \\
+\sum_{k=1}^{\rho} S\left(t-t_{k}\right) I_{k}\left(x\left(t_{k}^{-}\right)\right)
\end{gathered}
$$

and control given by following formula:

$$
\begin{gathered}
u(t)=B^{*} S^{*}(T-t) \mathbf{E}\left\{( \Gamma _ { 0 } ^ { T } ) ^ { - 1 } \left[x(T)-S(T) x_{0}\right.\right. \\
-\int_{0}^{T} S(T-s) F(s, x(s)) d s-\int_{0}^{T} S(t-s) \sigma(s, x) d W(s) \\
\left.\left.-\sum_{k=1}^{\rho} S\left(T-t_{k}\right) I_{k}\left(x\left(t_{k}^{-}\right)\right)\right] \mid \mathcal{F}_{t}\right\} .
\end{gathered}
$$

At the end, the authors confirm with the Banach fixed-point theorem that the system (14) is completely controllable if the operator $\Phi$ has a unique fixed point.

\subsection{Impulsive neutral functional evolution integro-} differential system. The special case of semilinear system is the impulsive neutral functional evolution integro-differential system given by the form:

$$
\begin{gathered}
\frac{d}{d t}\left[x(t)+g\left(t, x_{t}\right)\right]=A(t) x(t) \\
+\int_{0}^{t} G(t, s) x(s) d s+(B u)(t) \\
+f\left(t, x_{t}, \int_{0}^{t} h\left(t, s, x_{s}\right) d s\right), \\
t \in J, \quad t_{t}, \quad k=1,2, \ldots, m, \\
\Delta x\left(t_{k}\right)=I_{k}\left(x_{t_{k}^{-}}\right), \quad x_{0}=\phi \in \mathcal{B}_{h},
\end{gathered}
$$

where $\mathcal{B}_{h}$ is the abstract phase space which is defined:

$$
\begin{gathered}
\mathcal{B}_{h}=\{\psi:(-\infty, 0] \rightarrow X, \text { such that for any } c>0, \\
\left.\left.\psi\right|_{[-c, 0]} \in \mathcal{B} \text { and } \int_{\infty}^{0} h(s)\|\psi\|_{[c, 0]} d s<\infty\right\}
\end{gathered}
$$

and for any $b>0$, it can define:

$$
\mathcal{B}=\{\psi:[-b, 0] \rightarrow X \text { such that } \psi(t)
$$

$$
\text { is bounded and measurable\} }
$$

and equip the space $\mathcal{B}$ with the norm:

$$
\|\psi\|_{[-b, 0]}=\sup _{s \in[-b, 0]}|\psi(s)|, \text { for all } \psi \in \mathcal{B} .
$$

Moreover:

- the state $x(\cdot)$ takes values in the Hilbert space $X$ with norm $\|\cdot\|$;

- $x_{t}$ represents function $x_{t}:(-\infty, 0] \rightarrow X$ defined by $x_{t}(\theta)=x(t+\theta), \infty<\theta<0$ which belongs to $\mathcal{B}_{h}$;

- the control $u(\cdot)$ is given in $L^{2}(J, V)$;

- a Hilbert space of admissible control functions with $V$ as a Hilbert space and thereby $J=[0, b]$;

- $D=\{(t, s): 0 \leq s \leq t \leq b\}$;

- $A(t)$ and $G(t)$ are closed operators on $X$ with dense domain $D(A)$ which is independent of $t$;

- $B$ is a bounded linear operator from $V$ to $X$;

- the nonlinear operators $g: J \times \mathcal{B}_{h} \rightarrow X, h: D \times \mathcal{B}_{h} \rightarrow X$ and $f: J \times \mathcal{B}_{h} \times X \rightarrow X$ are continuous;

- $I_{k}: X \rightarrow X, 0=t_{0}<t_{1}<\ldots<t_{k}<t_{k+1}=b$.

Let

$\mathcal{P C}((-\infty, b], X)=\{x: x$ be a function from $(-\infty, b]$ into $X$ such that $x(t)$ is continuous at $t \neq t_{k}$ and left continuous

at $t=t_{k}$ and the right limit $x\left(t_{k}^{+}\right)$exists for $\left.k=1,2, \ldots, m\right\}$. Note, $\mathcal{P C}((\infty, b], X)$ is a Banach space with norm

$$
\|x\|_{\mathcal{P C}}=\sup _{t \in[0, b]}\|x(t)\| .
$$

Below, we introduce the definitions both of a mild solution and a reachable set of the impulsive neutral functional evolution integro-differential system.

Definition 8 [66]. A function $x(\cdot) \in \mathcal{P C}((-\infty, b], X)$ is said to be a mild solution of dynamical system (15) if the following hold:

$$
x_{0}=\phi \in \mathcal{B}_{h} \text { on }(-\infty, 0],\left.\quad \Delta x\right|_{t=t_{k}}, \quad k=1,2, \ldots, m ;
$$

the restriction of $x(\cdot)$ to the interval $J_{k}, k=0,1, \ldots, m$, is continuous; for each $t \in[0, b)$, the function $U(t, s) A(s) g\left(s, x_{s}\right), s \in[0, t)$ is integrable and the impulsive integral equation 
J. Klamka, A. Babiarz, and M. Niezabitowski

$$
\begin{aligned}
& x(t)= U(t, 0)[\phi(0)+g(0, \phi)]-g\left(t, x_{t}\right) \\
&-\int_{0}^{t} U(t, s) A(s) g\left(s, x_{s}\right) d s \\
&+\int_{0}^{t} U(t, s) \int_{0}^{s} G(s, \tau) x(\tau) d \tau d s \\
&+\int_{0}^{t} U(t, s) {\left[\int_{0} B(s)+f\left(s, x_{s}, \int_{0}^{s} h\left(s, \tau, x_{\tau}\right)\right] d s\right.} \\
&+\sum_{0<t_{k}<t} U\left(t, t_{k}\right) I_{k}\left(x\left(t_{k}^{-}\right)\right), \quad t \in J,
\end{aligned}
$$

is satisfied.

Definition 9 [66]. The reachable set for the system (15) is described by following formula:

$$
\mathcal{R}\left(b, x_{0}\right)=\left\{x_{b}\left(x_{0} ; u\right)(0): u(\cdot) \in L^{2}(J, V)\right\}
$$

with initial value $x_{0}=\phi \in \mathcal{B}_{h}$ and state value $x_{b}\left(x_{0} ; u\right)$ at terminal time $b$ corresponding to control $u$.

In order to examine complete controllability of (15), it should be assumed the following hypotheses [66].

Hypothesis 23. The function $g: J \times \mathcal{B}_{h} \rightarrow X$ is continuous and there exist constants $L_{g}>0, N_{g}>0$ such that:

$$
\left\|g\left(t, \phi_{1}\right)-g\left(s, \phi_{2}\right)\right\| \leq L_{g}\left[|t-s|+\left\|\phi_{1}-\phi_{2}\right\|_{\mathcal{B}_{h}}\right],
$$

for every $t, s \in J$ and $\phi_{1}, \phi_{2} \in \mathcal{B}_{h}$ and

$$
\begin{gathered}
\left\|A(t) g\left(s_{1}, \phi\right)-A(t)\left(s_{2}, \psi\right)\right\| \leq N_{g}\left[\left|s_{1}-s_{2}\right|+\|\phi-\psi\|_{\mathcal{B}_{h}}\right], \\
s_{1}, s_{2} \in J, \quad \phi, \psi \in \mathcal{B}_{h} .
\end{gathered}
$$

Hypothesis 24. $A(t)$ generates a strongly continuous semigroup of a family of evolution operators $U(t, s)$ and there exist positive constants $M_{1}>0, M_{2}>0$ such that $\|U(t, s)\| \leq M_{1}$ and $\|G(t, s)\| \leq M_{2}$.

Hypothesis 25. The linear operator $\Lambda$ from $L^{2}(J, U)$ into $X$ defined by:

$$
\Lambda u=\int_{0}^{b} U(b, s) B u(s) d s
$$

has an inverse operator $\Lambda^{-1}$ defined on $L^{2}(J, U) / \operatorname{Ker} \Lambda$ and there exists a constant $K_{\Lambda}>0$ such that $\left\|B \Lambda^{-1}\right\| \leq K_{\Lambda}$.

Hypothesis 26. The nonlinear functions $f$ and $h$ satisfy the Lipschitz condition and there exist constants $F_{A}>0, H_{A}>0$ such that

$$
\left\|f\left(t, x_{t}, u_{t}\right)-f\left(t, y_{t}, v_{t}\right)\right\| \leq F_{A}(\|x-y\|+\|u-v\|)
$$

for $x, y, u, v \in X, t \in J$,

$$
\int_{0}^{t}\left\|h\left(t, s, x_{s}\right)-h\left(t, s, y_{s}\right)\right\| d s \leq H_{A}\|x-y\|
$$

for $x, y \in X, t, s \in J$.
Hypothesis 27. $I_{k}: X \rightarrow X$ is continuous and there exist constants $l_{k}$ such that

$$
\left\|I_{k}(x)-I_{k}(y)\right\| \leq l_{k}\|x-y\|, \quad k=1,2, \ldots, m .
$$

for each $x, y \in X$.

Using Hypothesis 25, the control can be given by following form:

$$
\begin{gathered}
u(t)=\Lambda^{-1}\left[x_{1}-U(b, 0)[\phi(0)+g(0, \phi)]\right. \\
+g\left(b, x_{b}\right)+\int_{0}^{b} U(b, s) A(s) g\left(s, x_{s}\right) d s \\
-\int_{0}^{b} U(b, s) \int_{0}^{s} G(s, \tau) x(\tau) d \tau d s \\
-\int_{0}^{b} U(b, s) f\left(s, x_{s}, \int_{0}^{s} h\left(s, \tau, x_{\tau}\right) d \tau\right) d s \\
\left.-\sum_{k=1}^{m} U\left(b, t_{k}\right) I_{k}\left(x\left(t_{k}^{-}\right)\right)\right](t) .
\end{gathered}
$$

Then, the nonlinear operator $\mathcal{P}$ from $\mathcal{P C}((-\infty, b], X)$ to $\mathcal{P C}((-\infty, b], X)$ defined by

$$
\begin{aligned}
& (\mathcal{P} x)(t)=U(t, 0)[\phi(0)+g(0, \phi)]-g\left(t, x_{t}\right) \\
& -\int_{0}^{t} U(t, s) A(s) g\left(s, x_{s}\right) d s \\
& +\int_{0}^{t} U(t, s) \int_{0}^{s} G(s, \tau) x(\tau) d \tau d s \\
& +\int_{0}^{t} U(t, \eta) B \Lambda^{-1}\left[x_{1}-U(b, 0)[\phi(0)+g(0, \phi)]\right. \\
& +g\left(b, x_{b}\right)+\int_{0}^{b} U(b, s) A(s) g\left(s, x_{s}\right) d s \\
& -\int_{0}^{b} U(b, s) \int_{0}^{s} G(s, \tau) x(\tau) d \tau d s \\
& -\int_{0}^{b} U(b, s) f\left(s, x_{s}, \int_{0}^{s} h\left(s, \tau, x_{\tau}\right) d \tau\right) d s \\
& \left.-\sum_{k=1}^{m} U\left(b, t_{k}\right) I_{k}\left(x\left(t_{k}^{-}\right)\right)\right](\eta) d \eta \\
& +\int_{0}^{t} U(t, s) f\left(s, x_{s}, \int_{0}^{s} h\left(s, \tau, x_{\tau}\right) d \tau\right) d s \\
& +\sum_{0<t_{k}<t} U\left(t, t_{k}\right) I_{k}\left(x\left(t_{k}^{-}\right)\right), \quad t \in J,
\end{aligned}
$$

has a fixed point $x(\cdot)$ when the control defined by (17) is used. 


\section{Banach fixed-point theorem in semilinear controllability problems - a survey}

Definition 10 [66]. The dynamical system (15) is said to be complete controllable on the interval $J$, if for every initial function $\phi \in \mathcal{B}_{h}$ and $x_{b} \in X$, there exists a control $u \in L^{2}(J, U)$ such that the solution $x(\cdot)$ of (15) satisfies $x(b)=x_{b}$.

Theorem 8 [66]. If Hypotheses 23-27 are satisfied and

$$
\begin{aligned}
& \left(1+b M_{1} K_{\Lambda}\right)\left[L_{g}+b M_{1} N_{g}+b^{2} M_{1} M_{2}\right. \\
& \left.+b M_{1} F_{A}\left(1+H_{A}\right)+M_{1} \Sigma_{k+1}^{m} l_{k}\right]<1,
\end{aligned}
$$

then the dynamical system (15) is completely controllable.

The proof [66] is based on the Banach fixed-point theorem.

4.4. Nonlinear stochastic neutral impulsive systems. The notion of complete controllability for nonlinear stochastic neutral impulsive systems in finite-dimensional spaces is considered in paper [64]. In that paper the authors obtained, using the Banach fixed-point theorem, the sufficient conditions ensuring the complete controllability of the Ito nonlinear stochastic impulsive system given by the following formula:

$$
\begin{gathered}
d[x(t)-g(t, x(t))]=[A(t) x(t)+B(t) u(t) \\
+f(t, x(t))] d t+\sigma(t, x(t)) d W(t), \quad t \neq t_{k} \\
\Delta x\left(t_{k}\right)=I_{k}\left(t_{k}, x\left(t_{k}^{-}\right)\right), \\
t=t_{k}, \quad k=1,2, \ldots, \rho, \\
x\left(t_{0}\right)=x_{0}, \quad t_{0} \geq 0,
\end{gathered}
$$

where

- $A(t), B(t)$ are given $n \times n, n \times m$ continuous matrices;

- $x(t) \in R^{n}$ is the vector describing the instantaneous state of the stochastic system;

- $u(t) \in R^{m}$ is a control input to the stochastic dynamical system;

- $g:\left[t_{0}, T\right] \times R^{n} \rightarrow R^{n}$ is differentiable

- $\sigma:\left[t_{0}, T\right] \times R^{n} \rightarrow R^{n \times n}$;

- $I_{k}: \Sigma \rightarrow R^{n}, \Sigma \subset\left[t_{0}, T\right] \times R^{n}$,

$\bullet$

$$
\Delta x(t)=x\left(t^{+}\right)-x\left(t^{-}\right)
$$

where

$$
\begin{aligned}
& \lim _{h \rightarrow 0^{+}} x(t+h)=x\left(t^{+}\right), \quad \lim _{h \rightarrow 0^{+}} x(t-h)=x\left(t^{-}\right) \\
& \text {and } 0=t_{0}<t_{1}<\ldots<t_{\rho}<t_{\rho+1}=T, \\
& \qquad I_{k}\left(x\left(t_{k}^{-}\right)\right)=\left(I_{1 k}\left(x\left(t_{k}^{-}\right)\right), \ldots, I_{n k}\left(x\left(t_{k}^{-}\right)\right)\right)^{T}
\end{aligned}
$$

describes the impulsive perturbation of state $x$ at time $t_{k}$ and $x\left(t_{k}^{-}\right)=x\left(t_{k}\right), k=1,2, \ldots, \rho$. Last implies that the solution of the Itô nonlinear stochastic impulsive system (19) is left continuous at $t_{k}$. The solution of the system (19) in the interval $\left[t_{0}, T\right]$ is expressed by the solution of the following equation:

$$
\begin{gathered}
x(t)=\Phi\left(t, t_{0}\right)\left[x_{0}-g\left(t_{0}, x_{0}\right)\right]+g(t, x(t)) \\
+\int_{t_{0}}^{t} \Phi(t, s) B(s) u(s) d s+\int_{t_{0}}^{t} A(s) \Phi(t, s) g(s, x(s)) d s \\
+\int_{t_{0}}^{t} \Phi(t, s) f(s, x(s)) d s+\int_{t_{0}}^{t} \Phi(t, s) \sigma(s, x(s)) d W(s) \\
+\sum_{k=1}^{\rho} \Phi\left(t, t_{k}\right) I_{k}\left(t_{k}, x\left(t_{k}^{-}\right)\right),
\end{gathered}
$$

where $\Phi(t, s)$ is $n \times n$ transition matrix associated with matrix $A(t)$. Similarly as before, it is necessary to assume some hypotheses [64].

Hypothesis 28. The functions $f, g$ and $\sigma$ satisfy the following Lipschitz condition: there exist constants $L_{1}, L_{2}, \alpha_{k}>0$ for $x, y \in \mathbb{R}^{n}$ and $t \in\left[t_{0}, T\right]$ such that

$$
\begin{gathered}
\|f(t, x)-f(t, y)\|^{2}+\|\sigma(t, x)-\sigma(t, y)\|^{2} \leq L_{1}\|x-y\|^{2}, \\
\|g(t, x)-g(t, y)\|^{2} \leq L_{2}\|x-y\|^{2}, \\
\left\|I_{k}(t, x)-I_{k}(t, y)\right\|^{2} \leq \alpha_{k}\|x-y\|^{2}, \quad k=1,2, \ldots, \rho .
\end{gathered}
$$

Hypothesis 29. The functions $f, g$ and $\sigma$ are continuous and satisfy the usual linear growth condition i.e., there exist constants $K_{1}, K_{2}, \beta_{k}>0$ for $x \in \mathbb{R}^{n}$ and $t \in\left[t_{0}, T\right]$ such that

$$
\begin{gathered}
\|f(t, x)\|^{2}+\|\sigma(t, x)\|^{2} \leq K_{1}\left(1+\|x\|^{2}\right), \\
\|g(t, x)\|^{2} \leq K_{2}\left(1+\|x\|^{2}\right), \\
\left\|I_{k}(t, x)\right\|^{2} \leq \beta_{k}\left(1+\|x\|^{2}\right), \quad k=1,2, \ldots, \rho .
\end{gathered}
$$

In order to apply the Banach fixed-point theorem, the nonlinear operator $\mathcal{P}$ from $\mathcal{B}_{2}$ to $\mathcal{B}_{2}$ (Banach space) has to be defined as follows:

$$
\begin{gathered}
(\mathcal{P} x)(t)=\Phi\left(t, t_{0}\right)\left[x_{0}-g\left(t_{0}, x_{0}\right)\right]+g(t, x(t)) \\
+\int_{t_{0}}^{t} \Phi(t, s) B(s) u(s) d s+\int_{t_{0}}^{t} A(s) \Phi(t, s) g(s, x(s)) d s \\
+\int_{t_{0}}^{t} \Phi(t, s) f(s, x(s)) d s+\int_{t_{0}}^{t} \Phi(t, s) \sigma(s, x(s)) d W(s) \\
+\sum_{k=1}^{\rho} \Phi\left(t, t_{k}\right) I_{k}\left(t_{k}, x\left(t_{k}^{-}\right)\right)
\end{gathered}
$$


J. Klamka, A. Babiarz, and M. Niezabitowski

with control expressed by the following formula:

$$
\begin{gathered}
u(t)=B^{*} \Phi^{*}(T, t) \mathbf{E}\left\{( \Gamma _ { t _ { 0 } } ^ { T } ) ^ { - 1 } \left(x_{T}-\Phi(T, 0)\left[x_{0}-g\left(t_{0}, x_{0}\right)\right]\right.\right. \\
\quad-g(T, x(T))-\int_{t_{0}}^{T} A(s) \Phi(T, s) g(s, x(s)) d s \\
-\int_{t_{0}}^{T} \Phi(t, s) f(s, x(s)) d s-\int_{t_{0}}^{T} \Phi(T, s) \sigma(s, x(s)) d W(s) \\
\left.\left.\quad-\sum_{k=1}^{\rho} \Phi\left(T, t_{k}\right) I_{k}\left(t_{k}, x\left(t_{k}^{-}\right)\right)\right) \mid \mathcal{F}_{t}\right\} .
\end{gathered}
$$

Now, we formulate theorem on complete controllability of the Itô nonlinear stochastic impulsive system (19).

Theorem 9 [64]. Suppose that the Hypotheses 28 hold, $T$ and $t_{0}$ are sufficiently close and operator $\Gamma_{t_{0}}^{T}$ is invertible. Then the system (20) is completely controllable if the following inequality:

$$
\begin{gathered}
{\left[9 L_{2}+9 l_{1}\left(1+M l_{1} l_{3}\right)\left(1+l_{2}\right)\left(L_{1}+L_{2}+\rho \sum_{k=1}^{\rho} \alpha_{k}\right)\right]} \\
\cdot\left(1+T-t_{0}\right)\left(T-t_{0}\right)<1,
\end{gathered}
$$

where

$$
\begin{gathered}
M=\max \left\{\left\|\Gamma_{s}^{T}\right\|^{2}: s \in\left[t_{0}, T\right]\right\}, \\
l_{1}=\max \left\{\|\Phi(t, s)\|^{2}: t_{0} \leq s<t \leq T\right\}, \\
l_{2}=\max \left\{\|A(s)\|^{2}: s \in\left[t_{0}, T\right],\right. \\
l_{3}=\frac{1}{\gamma} \geq\left\|\left(\Gamma_{t_{0}}^{T}\right)^{-1}\right\| \quad \text { for some } \gamma>0
\end{gathered}
$$

is valid.

Using the Banach fixed-point theorem it can be proved that the nonlinear operator $\mathcal{P}$ has a fixed point in $\mathcal{B}_{2}$ and then the dynamical system (20) is completely controllable.

\section{Examples}

In this we present two examples concerning the complete controllability of the dynamical systems for the wave equation and nonlinear stochastic impulsive system.

Example 1 [78]. Let us focus on the controlled wave equation with a distributed control $u(t, \cdot) \in L_{2}(0,1)$ given by the following equation:

$$
\begin{gathered}
d\left(\frac{\partial z(t, \theta)}{\partial t}\right) \\
=\left[\frac{\partial^{2} z(t, \theta)}{\partial^{2}}+u(t, \theta)+f(t, z(t, \theta))\right] d t+d W(t), \\
z(t, 0)=z(t, 1)=0, \\
z(0, \theta)=f(\theta), \quad \frac{\partial}{\partial t} z(0, \theta)=g(\theta),
\end{gathered}
$$

where $W(\cdot)$ is one dimensional Wiener process. According to [47], we introduce the Hilbert space $H=D\left(A_{0}^{1 / 2}\right) \oplus L_{2}(0,1)$ with the inner product:

$$
\begin{gathered}
\langle w, v\rangle=\left\langle\left[\begin{array}{l}
w_{1} \\
w_{2}
\end{array}\right],\left[\begin{array}{l}
v_{1} \\
v_{2}
\end{array}\right]\right\rangle \\
=\sum_{n=1}^{\infty}\left\{n^{2} \pi^{2}\left\langle w_{1}, e_{n}\right\rangle\left\langle e_{n}, v_{1}\right\rangle+\left\langle w_{2}, e_{n}\right\rangle\left\langle e_{n}, v_{s}\right\rangle\right\},
\end{gathered}
$$

where $e_{n}(\theta)=\sqrt{2} \sin (n \pi \theta)$.

Fixing

$$
\begin{gathered}
x=\left[\begin{array}{c}
z \\
\frac{\partial z}{\partial t}
\end{array}\right], \quad x(0)=\left[\begin{array}{l}
f \\
g
\end{array}\right], \\
B=\left[\begin{array}{c}
0 \\
I
\end{array}\right], \quad D=\left[\begin{array}{l}
0 \\
I
\end{array}\right],
\end{gathered}
$$

the posed problem can be rewritten as follows:

$$
\begin{gathered}
d x(t)=(A x(t)+B u(t)+f(t, x(t)) d t+D d W, \\
x(0)=\left[\begin{array}{c}
f \\
g
\end{array}\right],
\end{gathered}
$$

where

$$
A=\left[\begin{array}{cc}
0 & I \\
-A_{0} & 0
\end{array}\right], \quad A_{0} h=-\left(\frac{d^{2}}{d \theta^{2}}\right) h
$$

with domain given by a formula:

$$
D\left(A_{0}\right)=\left\{h \in L_{2}(0,1) \mid h,\left(\frac{d}{d \theta}\right) h\right. \text { are absolutely }
$$

continuous $\left(\frac{d^{2}}{d \theta^{2}}\right) h \in L_{2}(0,1)$ and $\left.h(0)=0=h(1)\right\}$.

Moreover, $A$ is the infinitesimal generator of a contraction semigroup $S(t)$ on $X$ expressed by:

$$
\begin{gathered}
S(t)\left[\begin{array}{l}
x_{1} \\
x_{2}
\end{array}\right] \\
=\sum_{n=1}^{\infty}\left[\begin{array}{cc}
\cos (n \pi t) & (n \pi)^{-1} \sin (n \pi t) \\
-n \pi \sin (n \pi t) & \cos (n \pi t)
\end{array}\right]\left[\begin{array}{l}
x_{1}^{n} \\
x_{2}^{n}
\end{array}\right] e_{n} .
\end{gathered}
$$

It should be pointed out, if Hypothesis 14 holds and the linear system associated with (23) is completely controllable on all $[0, t], t>0$ then the system (23) is completely controllable on $[0, T]$ provided that $f$ satisfies Hypotheses 11 and 12 .

Example 2 [64]. Let us consider the nonlinear stochastic impulsive system described by the following equations: 


$$
\begin{aligned}
& d\left[x_{1}-\frac{1}{2} x_{2}\right] \\
& =\left[x_{1}+1.4 u_{1}+0.8 u_{2}+x_{1} \cos \left(x_{2}\right)-2 x_{2}\right] d t \\
& +2 t^{2} x_{1} e^{-t} d W_{1}(t), \\
& d\left[x_{2}-\cos \left(x_{1}\right)\right] \\
& =\left[x_{2}-0.6 u_{1}+u_{2}+x_{2} \sin \left(x_{1}\right)+3 x_{1}\right] d t \\
& +x_{2} e^{-t} d W_{2}(t) \text {, } \\
& {\left[\begin{array}{c}
\Delta x_{1}\left(t_{k}\right) \\
\Delta x_{2}\left(t_{k}\right)
\end{array}\right]=e^{-0.5 k}\left[\begin{array}{cc}
0.12 & 0.5 \\
-0.6 & 0.15
\end{array}\right]\left[\begin{array}{l}
x_{1}\left(t_{k}^{-}\right) \\
x_{2}\left(t_{k}^{-}\right)
\end{array}\right] \text {, }} \\
& t=t_{k}, \quad k=1,2, \ldots, \rho, \quad x(0)=x_{0},
\end{aligned}
$$

where $t_{k}=t_{k-1}+0.2$.

The above-mentioned dynamical system can be expressed in the following form:

$$
\begin{gathered}
A(t)=\left[\begin{array}{ll}
1 & 0 \\
0 & 1
\end{array}\right], \quad B(t)=\left[\begin{array}{cc}
1.4 & 0.8 \\
-0.6 & 1
\end{array}\right], \\
\Phi(t, 0)=\left[\begin{array}{ll}
e^{t} & 0 \\
0 & e^{t}
\end{array}\right], \quad g(t, x(t))=\left[\begin{array}{c}
\frac{1}{2} x_{2}(t) \\
\cos \left(x_{1}(t)\right)
\end{array}\right], \\
f(t, x(t))=\left[\begin{array}{cc}
x_{1}(t) \cos \left(x_{2}(t)\right)-2 x_{2}(t) \\
x_{2}(t) \sin \left(x_{1}(t)\right)+3 x_{1}(t)
\end{array}\right], \\
\sigma(t, x(t))=\left[\begin{array}{cc}
2 t^{2} x_{1}(t) e^{-t} & 0 \\
0 & x_{2}(t) e^{-t}
\end{array}\right]
\end{gathered}
$$

with assumption that $x(t)=\left(x_{1}(t), x_{2}(t)\right) \in \mathbb{R}^{2}, t_{0}=0$. Furthermore, the controllability matrix given by the form:

$$
\begin{aligned}
& \Gamma_{0}^{T}=\int_{0}^{T} \Phi(T, s) B(s) B^{*}(s) \Phi^{*}(T, s) d s \\
= & \int_{0}^{T}\left[\left[\begin{array}{cc}
e^{(T-s)} & 0 \\
0 & e^{(T-s)}
\end{array}\right] \cdot\left[\begin{array}{cc}
1.4 & 0.8 \\
-0.6 & 1
\end{array}\right]\right. \\
\cdot & {\left.\left[\begin{array}{cc}
1.4 & -0.6 \\
0.8 & 1
\end{array}\right] \cdot\left[\begin{array}{cc}
e^{(T-s)} & 0 \\
0 & e^{(T-s)}
\end{array}\right]\right] d s } \\
= & {\left[\begin{array}{cc}
1.3 e^{2 T}-1.3 & 0.02-0.02 e^{2 T} \\
0.02-0.02 e^{2 T} & 0.68 e^{2 T}-0.68
\end{array}\right] }
\end{aligned}
$$

is invertible if $T>0$.

Moreover, it should be noted that all hypotheses are satisfied, which implies that the system given by Eq. (24) is completely controllable on $[0, T]$.

\section{Conclusions}

The article has described the complete controllability of different types of dynamical systems in an infinite-dimensional space. In addition, the paper presents the results of the selected works (and the best works by authors' opinion) from the scope of the study complete controllability of nonlinear dynamical systems.

The sufficient conditions of complete controllability were derived using the Banach fixed-point theorem. In each considered kind of a dynamical system the appropriate hypotheses were used, necessary to prove the complete controllability using a nonlinear operator which has a fixed point.

Moreover, controllability problems for different types of dynamical systems require the application of various mathematical concepts and methods taken directly from differential geometry, functional analysis, topology, matrix analysis and theory of ordinary and partial differential equations, and theory of difference equations. It is worth to notice, that there are many open problems for controllability concepts for special types of dynamical systems. For example, to this day (according to the best authors' knowledge), the most researches on controllability problems have been mainly concerned with unconstrained controls and without delays in the state variables or in the controls.

Acknowledgements. The research presented here was done by the authors as parts of the projects funded by the National Science Centre in Poland granted according to decisions DEC-2012/07/B/ST7/01404, DEC-2014/13/B/ST7/00755 and DEC-2012/07/N/ST7/03236, respectively.

\section{REFERENCES}

[1] J. Klamka, Controllability of Dynamical Systems, Kluwer Academic, Dordrecht, 1991.

[2] J. Klamka, "Controllability of dynamical systems - a survey", Arch. Control Sci. 2 (3/4), 281-307 (1993).

[3] R.E. Kalman, "Contribution to the theory of optimal control", Bol. Soc. Mat. Mex. 2 (5), 102-119 (1960).

[4] H.O. Fattorini, "Some remarks on complete controllability", SIAM J. Control Optim. 4, 686-694 (1967).

[5] D.L. Russel, "Nonharmonic Fourier series in the controllability theory of distributed parameter systems", J. Math. Anal. Appl. 18, 542-560 (1967).

[6] P. Muthukumar and P. Balasubramaniam, "Approximate controllability of nonlinear stochastic evolution systems with timevarying delays", J. Franklin I. 346 (1), 65-80 (2009).

[7] D.N. Chalishajar, R.K. George, A.K. Nandakumaran, and F.S. Acharya, "Trajectory controllability of nonlinearintegrodifferential system", J. Franklin I. 347 (7), 1065-1075 (2010).

[8] J. Huang, Z. Han, X. Cai, and L. Liu, "Control of timedelayed linear differential inclusions with stochastic disturbance", J. Franklin I. 347 (10), 1895-1906 (2010).

[9] L. Tie, K. Cai, and Y. Lin, "On controllability of discrete-time bilinear systems", J. Franklin I. 348 (5), 933-940 (2011).

[10] Z. Yan, "Controllability of fractional-order partial neutral functional integro-differential inclusions with infinite delay", J. Franklin I. 348 (8), 2156-2173 (2011).

[11] P. Muthukumar and P. Balasubramaniam, "Approximate controllability of mixed stochastic Volterra-Fredholm type integrodifferential systems in Hilbert space”, J. Franklin I. 348 (10), 2911-2922 (2011).

[12] S. Yang, B. Shi, and Q. Zhang, "Complete controllability of nonlinear stochastic impulsive functional systems", Appl. Math. Comput. 218 (9), 5543-5551 (2012). 
[13] J. Klamka, "Controllability of dynamical systems. A survey", Bull. Pol. Ac.: Tech. 61 (2), 335-342 (2013).

[14] P. Muthukumar and C. Rajivganthi, "Approximate controllability of impulsive neutral stochastic functional differential system with state-dependent delay in Hilbert spaces", J. Control Theory Appl. 11 (3), 351-358 (2013).

[15] J. Klamka, A. Czornik, and M. Niezabitowski, "Stability and controllability of switched systems", Bull. Pol. Ac.: Tech. 61 (3), 547-555 (2013).

[16] V. Vijayakumara, C. Ravichandranb, R. Murugesuc, and J.J. Trujillod, "Controllability results for a class of fractional semilinear integro-differential inclusions via resolvent operators", Appl. Math. Comput. 247, 152-161 (2014).

[17] H. Qin, J. Liu, and X. Zuo, "Controllability problem for fractional integro-differential evolution systems of mixed type with the measure of noncompactness", J. Inequal. Appl. 292, CDROM (2014).

[18] Z. Yan and F. Lu, "On approximate controllability of fractional stochastic neutral integro-differential inclusions with infinite delay", Appl. Anal. 94 (6), 1235-1258 (2015).

[19] J. Klamka, "Constrained exact controllability of semilinear systems", Syst. Control Lett. 47 (2), 139-147 (2002).

[20] J. Klamka, "Relative controllability of nonlinear systems with distributed delays in control", Int. J. Control 28 (2), 307-312 (1978).

[21] J. Klamka, "Constrained controllability of nonlinear systems", J. Math. Anal. Appl. 201 (2), 365-374 (1996).

[22] J. Klamka, "Schauder's fixed-point theorem in nonlinear controllability problems", Control Cybern. 29 (1), 153-165 (2000).

[23] K. Balachandran and J. Dauer, "Controllability of nonlinear systems in Banach spaces: A survey", J. Optimiz. Theory App. 115 (1), 7-28 (2002).

[24] H.X. Zhou, "Approximate controllability for a class of semilinear abstract equations", SIAM J. Control Optim. 21 (4), 551565 (1983).

[25] K. Naito and J.Y. Park, "Approximate controllability for trajectories of a delay Volterra control system", J. Optimiz. Theory App. 61 (2), 271-279 (1989).

[26] R.K. George, "Approximate controllability of nonautonomous semilinear systems", Nonlinear Anal-Theor. 24 (9), 1377-1393 (1995).

[27] N.I. Mahmudov and S. Zorlu, "Approximate controllability of semilinear neutral systems in Hilbert spaces", J. App. Math. Stoch. Anal. 16 (3), 233-242 (2003).

[28] J. Klamka, "Constrained controllability of semilinear systems with multiple delays in control", Bull. Pol. Ac.: Tech. 52 (1), 25-30 (2004).

[29] J. Klamka, "Constrained controllability of semilinear systems with delayed controls", Bull. Pol. Ac.: Tech. 56 (4), 333-337 (2008).

[30] N.I. Mahmudov, "Approximate controllability of semilinear deterministic and stochastic evolution equations in abstract spaces", SIAM J. Control Optim. 42 (5), 1604-1622 (2003).

[31] N.I. Mahmudov and M.A. McKibben, "Abstract secondorder damped McKean-Vlasov stochastic evolution equations", Stoch. Anal. Appl. 24 (2), 303-328 (2006).

[32] J. Klamka, "Stochastic controllability and minimum energy control of systems with multiple delays in control", Appl. Math. Comput. 206 (2), 704-715 (2008).

[33] K. Balachandran and S. Karthikeyan, "Controllability of nonlinear Ito type stochastic integro-differential systems",
J. Franklin I. 345 (4), 382-391 (2008).

[34] J. Klamka, "Stochastic controllability of systems with variable delay in control", Bull. Pol. Ac.: Tech. 56 (3), 279-284 (2008).

[35] J. Klamka, "Constrained controllability of semilinear systems with delays", Nonlinear Dynam. 56 (1-2), 169-177 (2009).

[36] J. Klamka, "Stochastic controllability of systems with multiple delays in control", Int. J. Appl. Math. Comput. Sci. 19 (1), 39-47 (2009).

[37] J. Klamka and B. Sikora, "On constrained stochastic controllability of dynamical systems with multiple delays in control", Bull. Pol. Ac.: Tech. 60 (2), 301-306 (2012).

[38] M.A. Dubov and B.S. Mordukhovich, "Theory of controllability of linear stochastic systems", Diff. Equat+ 14, 1609-1612 (1978).

[39] J. Zabczyk, Controllability of stochastic linear systems, Syst. Control Lett. 1 (1), 25-31 (1981).

[40] M. Ehrhardt and W. Kliemann, "Controllability of stochastic linear systems", Syst. Control Lett. 2 (3), 145-153 (1982).

[41] N.I. Mahmudov and A. Denker, "On controllability of linear stochastic systems", Int. J. Control 73 (2), 144-151 (2000).

[42] N.I. Mahmudov, "Controllability of linear stochastic systems", IEEE T. Automat. Contr. 46 (5), 724-731 (2001).

[43] A. Czornik and A. Świerniak, "On direct controllability of discrete time jump linear system”, J. Franklin I. 341 (6), 491-503 (2004).

[44] A. Czornik and A. Świerniak, "Controllability of discrete time jump linear systems", Dynam. Cont. Dis. Ser. B 12 (2), 165189 (2005).

[45] A.E. Bashirov and N.I. Mahmudov, "On concepts of controllability for linear deterministic and stochastic systems", SIAM J. Control Optim. 37 (6), 1808-1821 (1999).

[46] N.I. Mahmudov, "Controllability of linear stochastic systems in Hilbert spaces", J. Math. Anal. Appl. 259 (1), 64-82 (2001).

[47] R.F. Curtain and H. Zwart, An Introduction to InfiniteDimensional Linear Systems Theory, 21, Springer Science \& Business Media, 1995.

[48] J. Zabczyk, Mathematical Control Theory: an Introduction, Springer Science \& Business Media, 2009.

[49] K. Naito, "Controllability of semilinear control systems dominated by the linear part", SIAM J. Control Optim. 25 (3), 715-722 (1987).

[50] V.N. Do, "A note on approximate controllability of semilinear systems", Syst. Control Lett. 12 (4), 365-371 (1989).

[51] M. Yamamoto and J.Y. Park, "Controllability for parabolic equations with uniformly bounded nonlinear terms", J. Optimiz. Theory App. 66 (3), 515-532 (1990).

[52] S.E. Mohammed, Stochastic Functional Differential Equations, Pitman, Boston, 1984.

[53] T. Taniguchi, "Almost sure exponential stability for stochastic partial functional differential equations", Stoch. Anal. Appl. 16 (5), 965-975 (1998).

[54] T. Taniguchi, K. Liu, and A. Truman, "Existence, uniqueness, and asymptotic behavior of mild solutions to stochastic functional differential equations in Hilbert spaces", J. Differ. Equations, 181 (1), 72-91 (2002).

[55] J. Wu, Theory and Applications of Partial Functional Differential Equations, Springer-Verlag, New York, 1996.

[56] J. Klamka, "Stochastic controllability of linear systems with state delays", Int. J. of Appl. Math. Comput. Sci. 17 (1), 5-13 (2007). 
[57] N.I. Mahmudov, "On controllability of semilinear stochastic systems in Hilbert spaces", IMA J. Math. Control I. 19 (4), 363-376 (2002).

[58] P. Balasubramaniam and J.P. Dauer, "Controllability of semilinear stochastic delay evolution equations in Hilbert spaces", Int. J. Math. Math. Sci. 31 (3), 157-166 (2002).

[59] X.Z. Liu and A.R. Willms, "Impulsive controllability of linear dynamical systems with applications to maneuvers of spacecraft", Math. Probl. Eng. 2 (4), 277-299 (1996).

[60] R.K. George, A.K. Nandakumaran, and A. Arapostathis, "A note on controllability of impulsive systems", J. Math. Anal. Appl. 241 (2), 276-283 (2000).

[61] S. Sivasundaram and J. Uvah, "Controllability of impulsive hybrid integro-differential systems", Nonlinear Anal. Hybrid Syst. 2 (4), 1003-1009 (2008).

[62] Z.H. Guan, T.H. Qian, and X. Yu, "Controllability and observability of linear time-varying impulsive systems", IEEE T. Circuits Syst. 49 (8), 1198-1208 (2002).

[63] Z.H. Guan, T.H. Qian, and X.H. Yu, "On controllability and observability for a class of impulsive systems", Syst. Control Lett. 47 (3), 247-257 (2002).

[64] S. Karthikeyan and K. Balachandran, "Controllability of nonlinear stochastic neutral impulsive systems", Nonlinear Anal. Hybrid Syst. 3 (3), 266-276 (2009).

[65] Y.K. Chang, "Controllability of impulsive functional differential systems with infinite delay in Banach spaces", Chaos Soliton. Fract. 33 (5), 1601-1609 (2007).

[66] B. Radhakrishnan and K. Balachandran, "Controllability of impulsive neutral functional evolution integro-differential systems with infinite delay", Nonlinear Anal. Hybrid Syst. 5 (4), 655 670 (2011)

[67] L. Shen, J. Shi, and J. Sun, "Complete controllability of impulsive stochastic integro-differential systems", Automatica 46 (6), 1068-1073 (2010).

[68] G.M. Xie and L. Wang, "Necessary and sufficient conditions for controllability and observability of switched impulsive control systems", IEEE T. Automat. Contr. 49 (6), 960-966 (2004).
[69] M.L. Li, M.S. Wang, and F.Q. Zhang, "Controllability of impulsive functional differential systems in Banach spaces", Chaos Soliton. Fract. 29 (1), 175-181 (2006).

[70] B. Liu and H.J. Marquez, "Controllability and observability for a class of controlled switching impulsive systems", IEEE T. Automat. Contr. 53 (10), 2360-2366 (2008).

[71] R. Sakthivel, N.I. Mahmudov, and J.H. Kim, "On controllability of second order nonlinear impulsive differential systems", Nonlinear Anal-Theor. 71 (1-2), 45-52 (2009).

[72] A. Pazy, Semigroups of Linear Operators and Applications to Partial Differential Equations, Springer-Verlag, New York, 1983.

[73] K. Balachandran and S. Karthikeyan, "Controllability of stochastic integro-differential systems", Int. J. Control 80 (3), 486-491 (2007).

[74] K. Balachandran, J.-H. Kim, and S. Karthikeyan, "Controllability of semilinear stochastic integro-differential equations", Kybernetika 43, 31-44 (2007).

[75] R. Subalakshmi, K. Balachandran, and J.Y. Park, "Controllability of semilinear stochastic functional integro-differential systems in Hilbert spaces", Nonlinear Anal. Hybrid Syst. 3 (1), 39-50 (2009).

[76] J.P. Dauer and N.I. Mahmudov, "Controllability of stochastic semilinear functional differential equations in Hilbert spaces", J. Math. Anal. Appl. 290 (2), 373-394 (2004).

[77] J.P. Dauer and N.I. Mahmudov, "Approximate controllability of semilinear functional equations in Hilbert spaces", J. Math. Anal. Appl. 273 (2), 310-327 (2002).

[78] N.I. Mahmudov, "Controllability of semilinear stochastic systems in Hilbert spaces", J. Math. Anal. Appl. 288 (1), 197-211 (2003).

[79] X. Dai and F. Yang, "Complete controllability of impulsive stochastic integro-differential systems in Hilbert space", Abstr. Appl. Anal. ID 783098, CD-ROM (2013).

[80] X.S. Dai and F.Q. Deng, "Controllability of semilinear stochastic impulsive systems in Hilbert spaces", Proc. Int. Con. Machine Learning and Cybernetics 6, 3657-3662 (2009). 